\title{
Diferenciação de pacientes cirróticos com e sem encefalopatia hepática a partir da análise de padrões motores finos: Um estudo piloto com o Leap Motion Controller
}

Differentiation of cirrhotic patients with and without hepatic encephalopathy from the analysis of

fine motor patterns: A pilot study with the Leap Motion Controller

Diferenciación de pacientes cirróticos con y sin encefalopatía hepática a partir del análisis de patrones de motricidad fina: Un estudio piloto con el Leap Motion Controller

Recebido: 02/06/2021 | Revisado: 13/06/2021 | Aceito: 16/06/2021 | Publicado: 30/06/2021

\author{
Manoel Miqueias Maia \\ ORCID: https://orcid.org/0000-0001-6712-0769 \\ Universidade Estadual do Ceará, Brasil \\ E-mail: miqueias.maia@aluno.uece.br \\ Francisco Sérgio Rangel de Paula Pessoa \\ ORCID: https://orcid.org/0000-0001-6025-5601 \\ Hospital Geral de Fortaleza, Brasil \\ E-mail: sergio_pessoa@uol.com.br \\ Cássio Pinheiro Oliveira \\ ORCID: https://orcid.org/0000-0001-8004-8087 \\ Fundação Oswaldo Cruz, Brasil \\ E-mail: cassiopo7@gmail.com \\ Paulo Henrique Pessoa Nobre \\ ORCID: https://orcid.org/0000-0002-7693-8607 \\ Universidade Federal do Ceará, Brasil \\ E-mail: paulohenriquepnobre@gmail.com \\ Cristiane Clemente de Mello Salgueiro \\ ORCID: https://orcid.org/0000-0003-0071-4030 \\ Universidade Estadual do Ceará, Brasil \\ E-mail: cristiane.mello@uece.br
}

\begin{abstract}
Resumo
Objetivo: analisar a precisão motora de pacientes cirróticos com ou sem encefalopatia hepática (EH), em diferentes gravidades, por meio da captura geoespacial das mãos. Metodologia: O público-alvo foram os pacientes do Serviço de Gastroenterologia de um hospital terciário do Nordeste do Brasil. Os dados foram coletados em três grupos de pacientes (A, EH não identificado; B, EH grau I e; C, EH grau II). A coleta dos dados de motricidade foi realizada com o Leap Motion Controller (LMC). Os dados coletados foram compostos pela posição de 16 pontos das mãos em três dimensões que, em sequência, foram convertidas em distância entre dois pontos. Resultados: Foram incluídos 60 pacientes com idade média de 54,6 ( \pm 14,7) anos. Os testes de Kruskal-Wallis e Dunn indicaram diferenças nas medianas das variáveis para os três grupos $(\mathrm{p}<0,05)$. As representações gráficas mostram uma diferença na precisão motora entre os grupos em um índice de $100 \%$ das variáveis, com variações com tendência de C > B $>$ A em $87,5 \%$ dos casos. As frequências de movimento dos dedos, de ambas as mãos, tiveram o potencial de diferenciar os grupos. A direção é mais discriminativa do que a posição e a velocidade. Conclusão: Os resultados sugerem a possibilidade de diferenciar as classes de pacientes e que a progressão do desvio motor é uma das complicações do agravamento da doença.
\end{abstract}

Palavras-chave: Encefalopatia hepática; Movimento; Projetos piloto.

\begin{abstract}
Aim: analyze the motor precision of cirrhotic patients with or without hepatic encephalopathy (HE), in different severities, through the geospatial capture of the hands. Methodology: The target audience was patients at the Gastroenterology Service of a tertiary hospital in Northeastern Brazil. Data were collected from three groups of patients (A, unidentified EH; B, grade I EH and; C, grade II EH). Motricity data collection was performed with the Leap Motion Controller (LMC). The collected data were composed by the position of 16 points of the hands in three dimensions that, in sequence, were converted into distance between two points. Results: 60 patients with a mean age of $54.6( \pm 14.7)$ years were included. The Kruskal-Wallis and Dunn tests indicated differences in the medians of the variables for the three groups $(\mathrm{p}<0.05)$. The graphical representations show a difference in motor precision between the groups in an index of $100 \%$ of the variables, with variations with a tendency of $\mathrm{C}>\mathrm{B}>\mathrm{A}$ in $87.5 \%$ of the cases. The frequency of movement of the fingers, of both hands, had the potential to differentiate the groups. Direction is
\end{abstract}


more discriminating than position and speed. Conclusion: The results suggest the possibility of differentiating the classes of patients and that the progression of motor deviation is one of the complications of the worsening of the disease.

Keywords: Hepatic encephalopathy; Movement; Pilot projects.

\section{Resumen}

Objetivo: analizar la precisión motora de pacientes cirróticos con o sin encefalopatía hepática (EH), en diferentes severidades, a través de la captura geoespacial de las manos. Metodología: El público objetivo fueron los pacientes del Servicio de Gastroenterología de un hospital terciario del Nordeste de Brasil. Se recogieron datos de tres grupos de pacientes (A, EH no identificado; B, EH grado I y; C, EH grado II). La recolección de datos de motricidad se realizó con el Leap Motion Controller (LMC). Los datos recolectados fueron compuestos por la posición de 16 puntos de las manos en tres dimensiones que, en secuencia, se convirtieron en distancia entre dos puntos. Resultados: se incluyeron 60 pacientes con una edad media de 54,6 ( \pm 14,7) años. Las pruebas de Kruskal-Wallis y Dunn indicaron diferencias en las medianas de las variables para los tres grupos $(\mathrm{p}<0.05)$. Las representaciones gráficas muestran una diferencia en la precisión motora entre los grupos en un índice del $100 \%$ de las variables, con variaciones con tendencia de C> B> A en el 87,5\% de los casos. La frecuencia de movimiento de los dedos, de ambas manos, tenía el potencial de diferenciar los grupos. La dirección discrimina más que la posición y la velocidad. Conclusión: Los resultados sugieren la posibilidad de diferenciar las clases de pacientes y que la progresión de la desviación motora es una de las complicaciones del agravamiento de la enfermedad.

Palabras clave: Encefalopatía hepática; Movimiento; Proyecto de piloto.

\section{Introdução}

A encefalopatia hepática $(\mathrm{EH})$ é um distúrbio no sistema nervoso central (SNC) relacionado com a disfunção hepática e/ou shunt portossistêmico, sendo uma das condições mais comuns da evolução da cirrose hepática (Amodio, 2018; Vilstrup et al., 2014), possuindo impacto negativo na sobrevida desses pacientes, influindo na redução da funcionalidade global, orientação em tempo e espaço, alterações no ciclo sono-vigília, habilidade motora e em outras funções neurocognitivas (Bajaj et al., 2008; Sotil et al., 2009). Trata-se de uma das formas de descompensação da cirrose mais comuns, associada a hemorragias digestivas, infecções, disfunção renal e, principalmente, a piora da ascite (Bittencourt et al., 2011). É tida como a segunda principal causa de internamento de cirróticos, com uma sobrevida mediana de 1-2 anos após seu desenvolvimento (Wijdicks, 2016).

Na classificação da EH quanto à gravidade, o sistema mais utilizado é o de West-Haven (Vilstrup et al., 2014), que distingue os estágios, a consciência, o intelecto e o comportamento do paciente, assim como os achados neurológicos em 5 gradações: mínima, grau I, II, III e IV. Os sinais da doença podem ser desde déficits subclínicos - como leves desvios motores -, podendo progredir até o estágio do coma (Bittencourt et al., 2011; Pinho, Cerqueira \& Peixoto, 2011; Pires \& Marinho, 2016), possuindo um amplo espectro de comprometimento mental. O Quadro 1 apresenta as características de consciência, cognição e comportamento e função neuromuscular, de acordo com cada uma das fases identificadas na classificação. 
Quadro 1. Classificação da encefalopatia hepática por gradações neuropsíquicas, segundo os Critérios de West-Haven.

\begin{tabular}{|c|c|l|l|}
\hline $\begin{array}{c}\text { Estágio / } \\
\text { Grau }\end{array}$ & Consciência & \multicolumn{1}{|c|}{ Cognição e comportamento } & \multicolumn{1}{|c|}{ Função neuromuscular } \\
\hline 0 (mínima) & Normal & $\begin{array}{l}\text { Perda assintomática de habilidades } \\
\text { cognitivas }\end{array}$ & $\begin{array}{l}\text { Exame normal, testes psicomotores } \\
\text { prejudicados }\end{array}$ \\
\hline I & $\begin{array}{c}\text { Leve perda de } \\
\text { atenção }\end{array}$ & $\begin{array}{l}\text { Distúrbios do sono, dificuldade de } \\
\text { concentração, depressão, ansiedade ou } \\
\text { irritabilidade e redução da atenção }\end{array}$ & $\begin{array}{l}\text { Voz monotônica, tremor, dificuldade na escrita, } \\
\text { apraxia de construção }{ }^{1} \text {, tremor ou flapping/leve }\end{array}$ \\
\hline II & Letárgico & $\begin{array}{l}\text { Sonolência discreta, desorientação, perda } \\
\text { da memória de curto-prazo, desinibição } \\
\text { no comportamento }\end{array}$ & $\begin{array}{l}\text { Flapping evidente, fala arrastada, ataxia }^{2}, \\
\text { disartria }^{3} \text {, automatismos (bocejar, piscar, sugar) }\end{array}$ \\
\hline III & $\begin{array}{l}\text { Sonolento, mas } \\
\text { responsivo }\end{array}$ & $\begin{array}{l}\text { Desorientação severa, sonolência intensa, } \\
\text { confusão, amnésia, raiva, paranoia ou } \\
\text { outros comportamentos bizarros }\end{array}$ & $\begin{array}{l}\text { Rigidez muscula e clônus }{ }^{4}, \text { hiperreflexia }{ }^{5}, \\
\text { nistagmo }\end{array}$ \\
\hline IV & Coma & Coma & $\begin{array}{l}\text { Pupilas dilatadas, reflexos oculoencefálico ou } \\
\text { oculovestibular, postura de descerebração }\end{array}$ \\
\hline
\end{tabular}

Fonte: Adaptado de <www.msdmanuals.com/pt/profissional/dist\%C3\%BArbios-hep\%C3\%A1ticos-e-biliares/abordagem-ao-paciente-comdoen\%C3\%A7a-hep\%C3\%A1tica/encefalopatia-portossist\%C3\%AAmica> e Vilstrup et al., 2014.

Clinicamente, um importante marcador da doença é a presença do flapping/asterixis, que se constitui em um movimento involuntário e grosseiro das mãos quando mantidos em hiperextensão, originando-se no punho (Agarwal \& Baid, 2016; Campagna et al., 2017). Segundo Amodio (2018), hoje este sinal clínico é fundamental na identificação do acometimento cerebral em decorrência de doença hepática.

Por afetar o SNC, existem formas de diagnóstico deste distúrbio que ocorrem por meio de atividades básicas correlatas à motricidade, tais como fazer liga-pontos, desenhos, pinturas, assinatura e a correlação do desempenho afetado na execução das tarefas (Nardone et al., 2016). À medida que o tratamento da doença progride, a execução destas atividades tem evolução positiva. Este processo depende de um acompanhamento muito próximo com o profissional que trata a patologia (Nardone et al., 2016).

Em detrimento dos achados literários acerca da análise da motricidade para a identificação da EH, o objetivo do desenvolvimento deste trabalho foi analisar e diferenciar a precisão motora de pacientes cirróticos, em diferentes gravidades, por meio da captura geoespacial das mãos. Este processo de captura geoespacial das mãos corresponde a utilização de um aparato tecnológico, composto por hardware e software, que observa e obtêm informações da posição de determinados pontos das mãos em três dimensões.

Para este fim, o processo foi realizado utilizando o Leap Motion Controller (LMC), um dispositivo de interação humano-computador e visão computacional com três sensores ópticos, duas câmeras e infravermelho, focado em capturar os movimentos das mãos (Dzikri \& Kurniawan, 2018), capaz de acompanhar comprimentos de ondas de luz em até $850 \mathrm{~nm}$.

\section{Metodologia}

Este estudo piloto e experimental teve como público-alvo os pacientes atendidos pelo Serviço de Gastroenterologia do Hospital Geral de Fortaleza (HGF), Fortaleza, Ceará, Brasil. Sua abordagem exploratória centra-se na finalidade de aquisição de hipóteses, por meio de análise estatística de dados, quanto à utilização de aparato tecnológico de hardware e software para

\footnotetext{
${ }^{1}$ Incapacidade de desenhar figuras geométricas.

${ }^{2}$ Equilíbrio ou coordenação motora prejudicados, possivelmente devido a danos no cérebro, nos nervos ou nos músculos.

${ }^{3}$ Fraqueza nos músculos usados para fala, o que muitas vezes faz com que a fala fique arrastada ou lenta.

${ }^{4}$ Contrações musculares involuntárias devido a um estiramento súbito do músculo.

${ }^{5}$ Condição neurológica caracterizada por atividade aumentada dos reflexos.

${ }^{6}$ Movimento involuntário dos olhos que pode fazer o olho mover-se rapidamente de um lado para outro, para cima e para baixo ou em um círculo, podendo borrar ligeiramente a visão.
} 
formulação futura de novos produtos ou testes diagnósticos, abrindo campo para novas explorações e validações (Piovesan \& Temporini, 1995; Casula, Rangarajan \& Shields, 2020).

Em respeito aos procedimentos éticos, o Comitê de Ética em Pesquisa da Universidade Estadual do Ceará aprovou esta pesquisa por meio do Certificado de Apresentação de Apreciação Ética no 36057420.3.0000.5534. Todos os participantes consentiram em participar assinando o Termo de Consentimento Livre e Esclarecido (TCLE).

\subsection{Grupos e critérios de inclusão e exclusão}

Foram coletados dados de três grupos clínicos de pacientes, de acordo com os Critérios de West-Haven (exceto mínima e graus III e IV), sendo divididos em: (A) pacientes cirróticos sem EH evidente (com ou sem EH mínima); (B) pacientes cirróticos com EH grau I - EH1; e (C) pacientes cirróticos com EH grau II - EH2. O recrutamento dos pacientes da pesquisa se deu por demanda espontânea nos ambulatórios ou a admissão na enfermaria.

Foram incluídos pacientes de ambos os sexos e maiores de 18 anos. Foram excluídos pacientes em estágio de coma (West-Haven grau IV); que não desejaram participar; não concordaram com os termos do TCLE; os que no momento da coleta de dados estavam em condições de saúde que impossibilitaram participar do estudo; os que possuíam outras desordens motoras que não causadas diagnosticamente pela EH, de acordo com os prontuários médicos e; os pacientes que, porventura, possuíam amputação nos membros superiores, bem como os usuários de medicações que reconhecidamente causam tremores.

\subsection{Coleta de dados}

A coleta de dados foi realizada em 15 aplicações no período de 21/09/2020 a 10/12/2020 em três ambulatórios e na enfermaria de gastroenterologia. A classificação de West-Haven $\left(y_{k}\right)$ foi obtida dos prontuários médicos.

Para a coleta dos dados de motricidade foi utilizado LMC, estando as mãos sobrepostas ao dispositivo e o paciente com olhos fechados e em pé. Os dados foram coletados, em média, três vezes, durante 10 segundos, com um minuto de intervalo entre uma coleta e outra para não causar fadiga muscular que prejudicasse a condição motora. $\mathrm{O}$ armazenamento dos dados foi realizado por meio do software "HandC", desenvolvido para esta atividade específica.

Os dados coletados pelo LMC foram compostos da posição dos 10 dedos e das duas palmas das mãos, a direção das palmas das mãos e a velocidade de movimentação em três dimensões $\left(x, y\right.$ e $z$ ), criando um vetor $\left(x_{k}\right)$ de 16 índices. Foram capturados 60 frames por segundo (fps), totalizando 28.800 entradas de dados em cada aplicação. A figura 1 apresenta o esquema de coleta dos pontos das mãos. 
Figura 1 - Esquema de coleta dos pontos das mãos. s: Leap Motion Controller. D1: polegar direito. D2 indicador direito. D3: dedo médio direito. D4: dedo anelar direito. D5: dedo mínimo direito. Dd: direção da mão direita. Dp: posição da palma da mão direita. Dv: velocidade da mão direita. E1: polegar esquerdo. E2: indicador esquerdo. E3: dedo médio esquerdo. E4: dedo anelar esquerdo. E5: dedo mínimo esquerdo. Ed: direção da mão esquerda. Ep: posição da palma da mão esquerda. Ev: velocidade da mão esquerda.

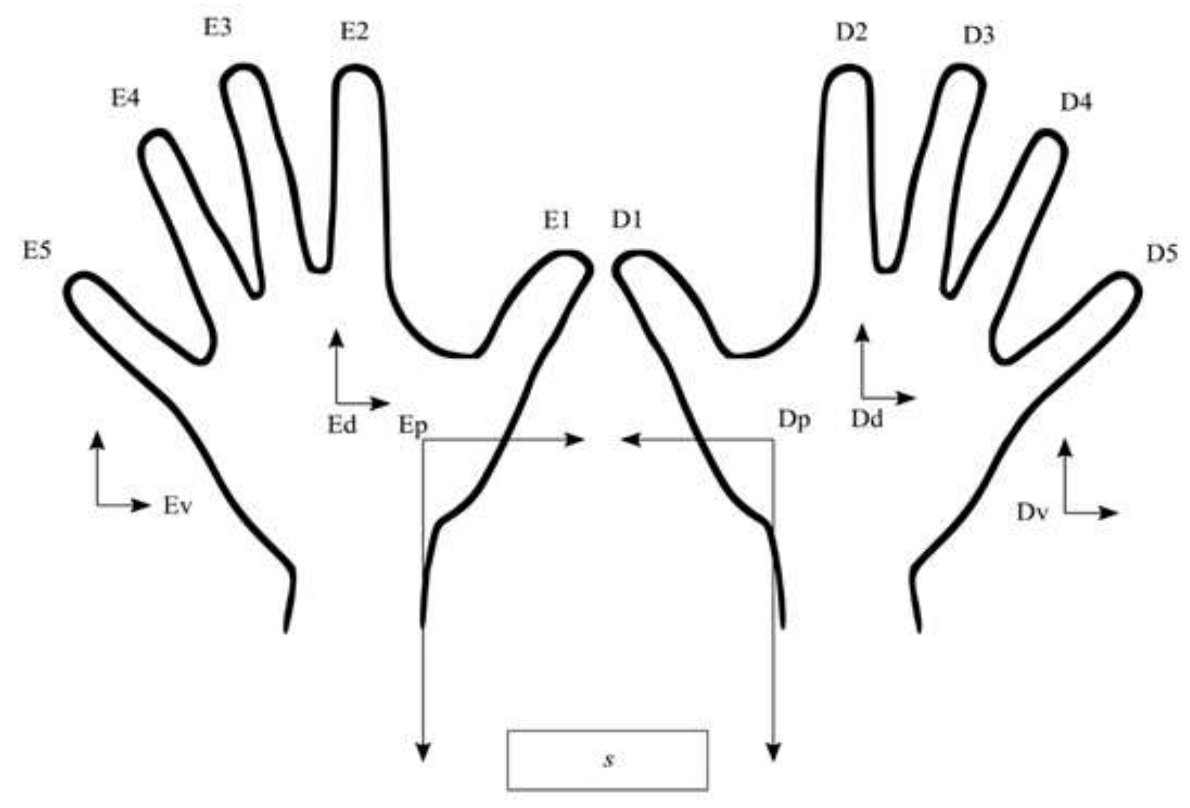

Fonte: Autores (2020).

\subsection{Processamento dos dados e análise}

Os valores tridimensionais dos pontos das mãos foram convertidos em distância entre dois pontos (dAB), conforme a equação 1.

Equação 1 - Distância entre dois pontos tridimensionais (dAB)

$$
d_{A B}=\sqrt{\left(x_{b}-x_{a}\right)^{2}+\left(y_{b}-y_{a}\right)^{2}+\left(z_{b}-z_{a}\right)^{2}}
$$

Cada vetor de dados, que corresponde a uma coleta de 10 segundos, foi tratado como um paciente, considerando que os dados da motricidade não são idênticos em cada aplicação. Cada conjunto de dados de uma observação foi associado a uma classificação dos critérios de West-Haven. Portanto, cada conjunto de dados de um paciente foi dada por um par ordenado $\left(x_{k}\right.$, $y_{k}$ ), composto por atributos (dados de movimentação) e classe (gravidade).

Objetivando reduzir o impacto da adição e remoção de mãos no início e no fim das capturas, foram eliminados 50 frames de cada aresta dos vetores, finalizando cada coleta com 500 frames válidos.

A análise dos dados foi realizada no RStudio, que é um ambiente integrado de desenvolvimento para a linguagem de programação $\mathrm{R}$, focado em computação estatística e visualização de dados. A priori foi analisada a normalidade dos dados da motricidade fina com o teste de Shapiro-Wilk, que é um teste de normalidade nas estatísticas frequentistas que resulta em uma hipótese nula (dados normalmente distribuídos) ou alternativa (dados anormalmente distribuídos). Posteriormente, as amostras independentes foram analisadas com o teste de Kruskal-Wallis, que é um método não paramétrico para testar se as amostras originam a mesma distribuição, utilizado para comparar duas ou mais amostras independentes; e, por fim, com o teste post-hoc de Dunn com correção de Bonferroni, que identifica quais medidas estatísticas são significativas das outras com redução da taxa de erro. Os gráficos em caixa também foram obtidos no RStudio.

Os dados foram balanceados com a técnica de super-amostragem sintética da minoria (SMOTE), que gera dados 
sintéticos para o balanceamento de classes de acordo com $k$-vizinhos próximos, conforme representado pela Figura 2.

Figura 2 - Discrição em diagrama da técnica SMOTE para a geração de dados sintéticos a partir de $k$-vizinhos próximos.

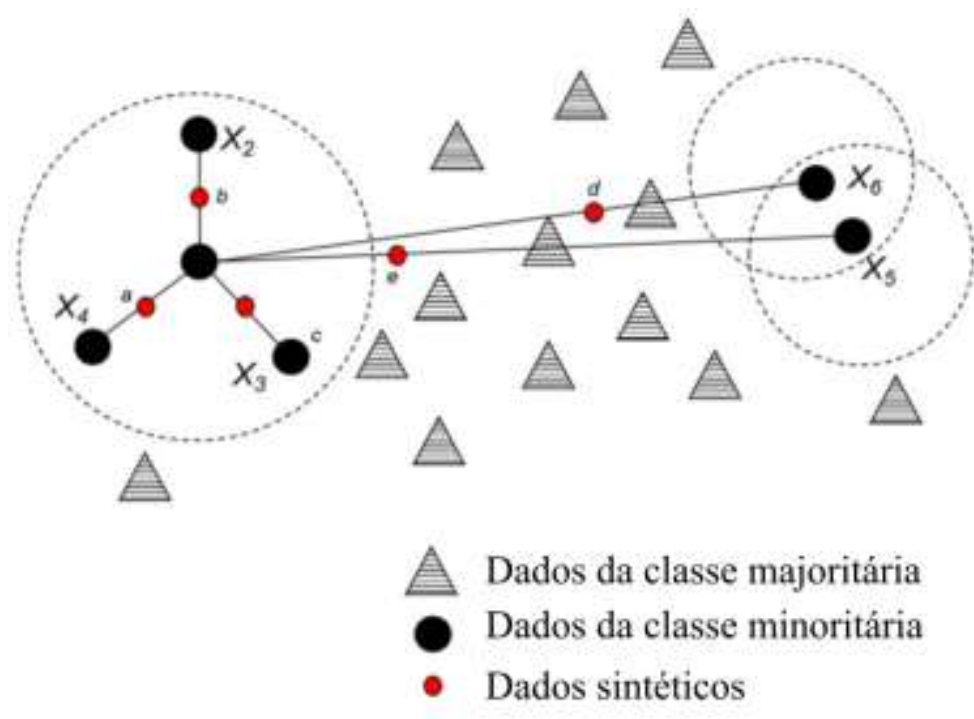

Fonte: Adaptado de Teh et al. (2020).

Como exemplificado na Figura 2, a técnica utilizada pelo SMOTE calcula a distância entre as propriedades do conjunto de dados e gera novas variáveis em seus pontos intermediários até atingir o quantitativo igualitário entre as classes ou grupos.

\section{Resultados}

Foram incluídos neste estudo 60 pacientes do Serviço de Gastroenterologia do HGF. A população da pesquisa foi composta por 44 indivíduos do sexo masculino e 16 do sexo feminino. A idade média dos indivíduos foi $54,1( \pm 14,7)$ anos, havendo ocorrências nas faixas etárias de 20-29 e 70-79 anos em ambos os grupos. A mediana da idade foi de 56 anos. A distribuição dos indivíduos no teste de qui-quadrado teve significância estatística $p<0,05$.

Quanto ao agravamento da cirrose por EH, 45 participantes não possuíam sinais evidentes (grupo A), podendo estar ou não em EH mínima; 10 possuíam sinais de EH grau I (grupo B) e 5 possuíam sinais de EH grau II (grupo C). Não foram obtidos dados de pacientes em EH grau III devido às incapacidades físicas dos indivíduos. As capturas de cada grupo foram A $=140 ; \mathrm{B}=24 ; \mathrm{C}=17$. Com a aplicação da técnica de SMOTE, as classes foram balanceadas igualmente para 140 amostras.

O teste de Shapiro-Wilk indicou ausência de distribuição normal e homogênea $(p<0,05)$ nos dados. Os testes de Kruskal-Wallis e Dunn indicaram diferenças das medianas das variáveis para os três grupos $(p<0,05)$.

Os gráficos de caixa representados pelas Figuras 3-7 apresentam os dados da precisão motora para cada uma das variáveis motoras coletadas. A apresentação está disposta de acordo com a identificação de cada dedo e das mãos por grupo.

A critério de compreensão, nos gráficos a mediana é representada pela linha central no retângulo (correspondente a $50 \%$ dos dados). A dispersão dos valores pode ser observada no espaço interquartílico entre o primeiro e o terceiro quartil, respectivamente $25 \%$ e $75 \%$ da concentração total dos dados. As caudas representam o comprimento entre os valores mínimos e máximos. Os valores além das caldas representam "outliers". 
Figura 3 - Amplitude da distância entre dois pontos tridimensionais, em milímetros (mm), dos dedos da mão direita por grupo. D1: polegar direito. D2 indicador direito. D3: dedo médio direito. D4: dedo anelar direito. D5: dedo mínimo direito.

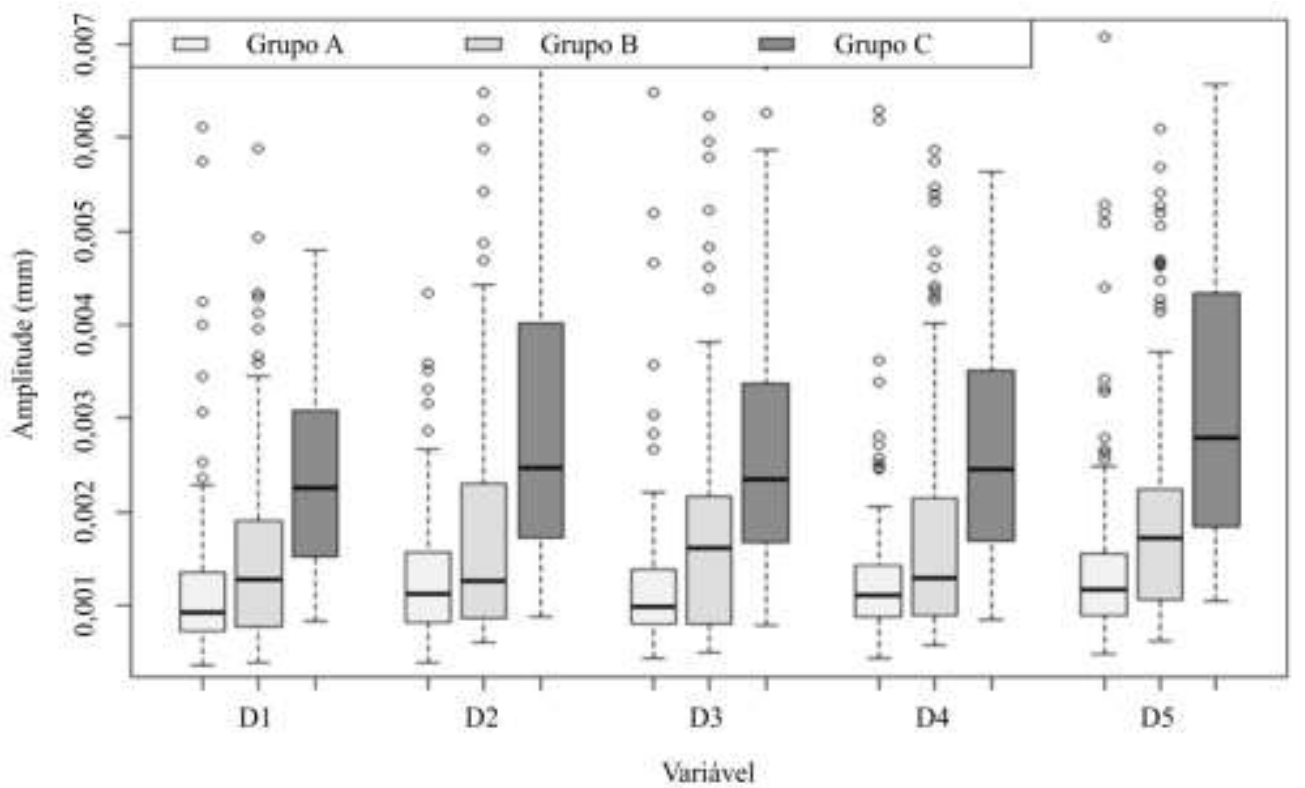

Fonte: Autores (2021).

Figura 4 - Amplitude da distância entre dois pontos tridimensionais, em milímetros (mm), dos dedos da mão esquerda por grupo. E1: polegar esquerdo. E2: indicador esquerdo. E3: dedo médio esquerdo. E4: dedo anelar esquerdo. E5: dedo mínimo esquerdo.

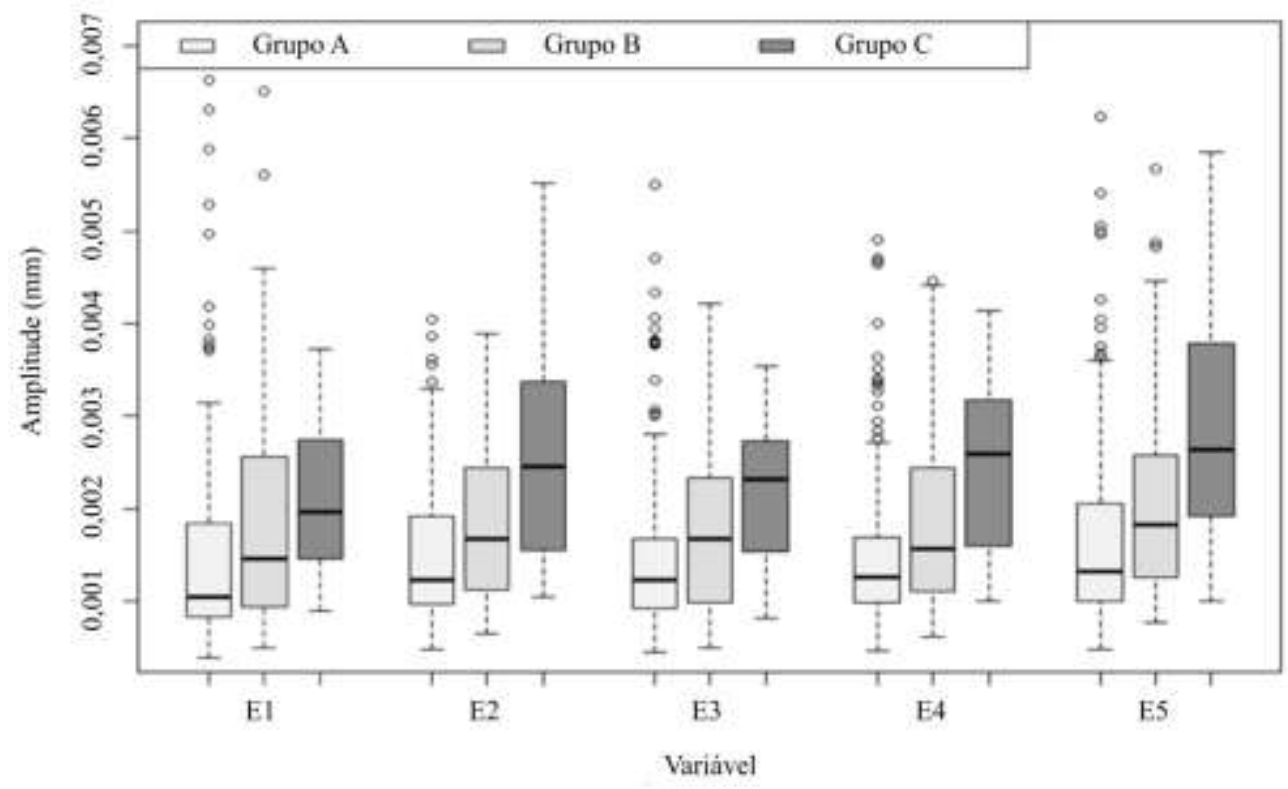

Fonte: Autores (2021). 
Research, Society and Development, v. 10, n. 7, e48310716749, 2021

(CC BY 4.0) | ISSN 2525-3409 | DOI: http://dx.doi.org/10.33448/rsd-v10i7.16749

Figura 5 - Amplitude da distância entre dois pontos tridimensionais, em milímetros (mm), da direção das mãos direita e esquerda por grupo. Dd: direção da mão direita. Ed: direção da mão esquerda.

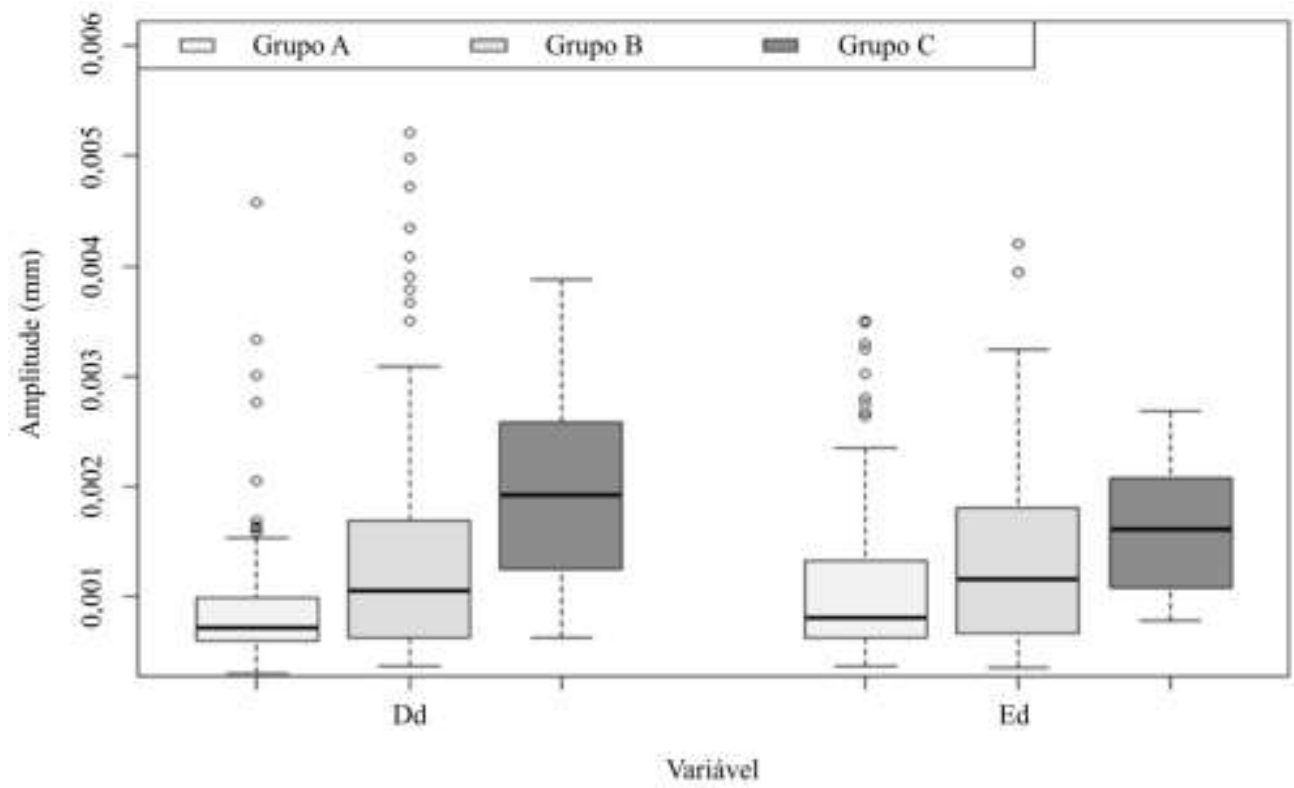

Fonte: Autores (2021).

Figura 6 - Amplitude da distância entre dois pontos tridimensionais, em milímetros (mm), da posição das mãos direita e esquerda por grupo. Dp: posição da mão direita. Ep: posição da mão esquerda.

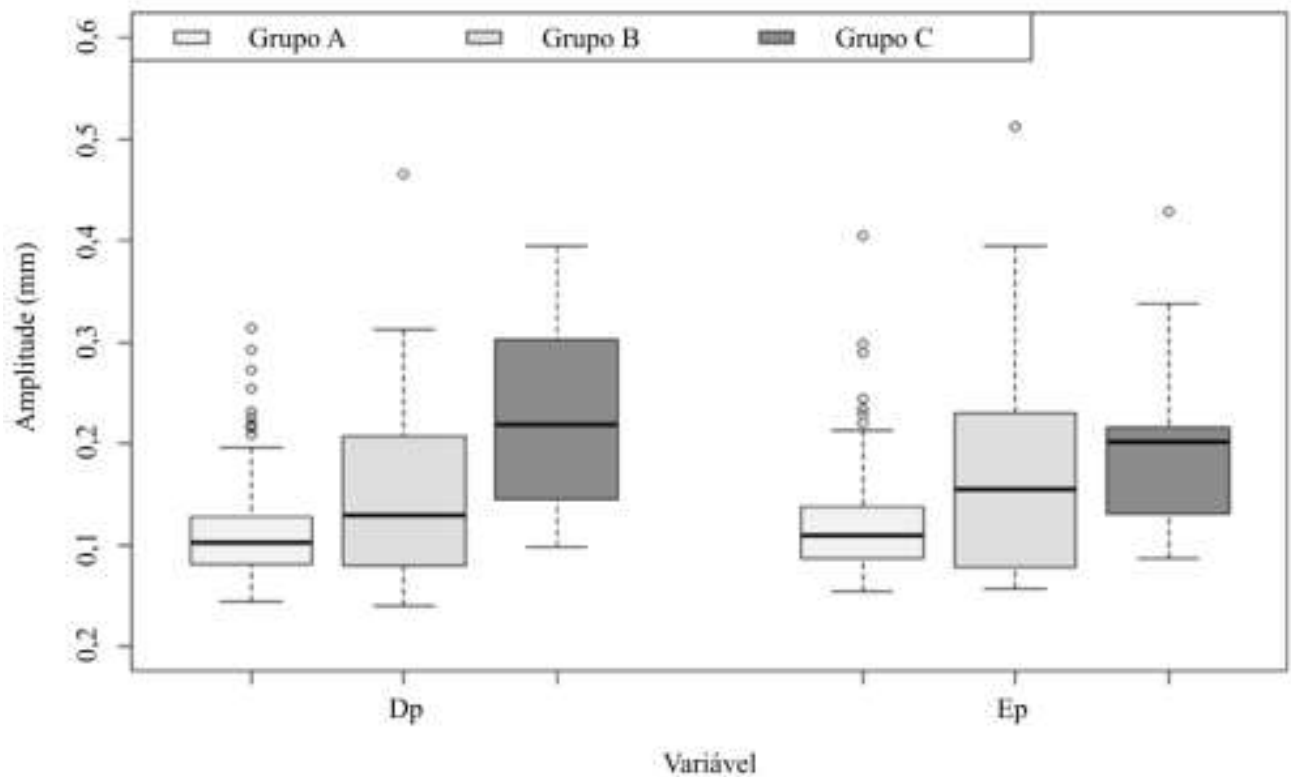

Fonte: Autores (2021). 
Figura 7 - Amplitude da distância entre dois pontos tridimensionais, em milímetros por segundo (mm/s), da velocidade das mãos direita e esquerda por grupo. Dv: velocidade da mão direita. Ev: velocidade da mão esquerda.

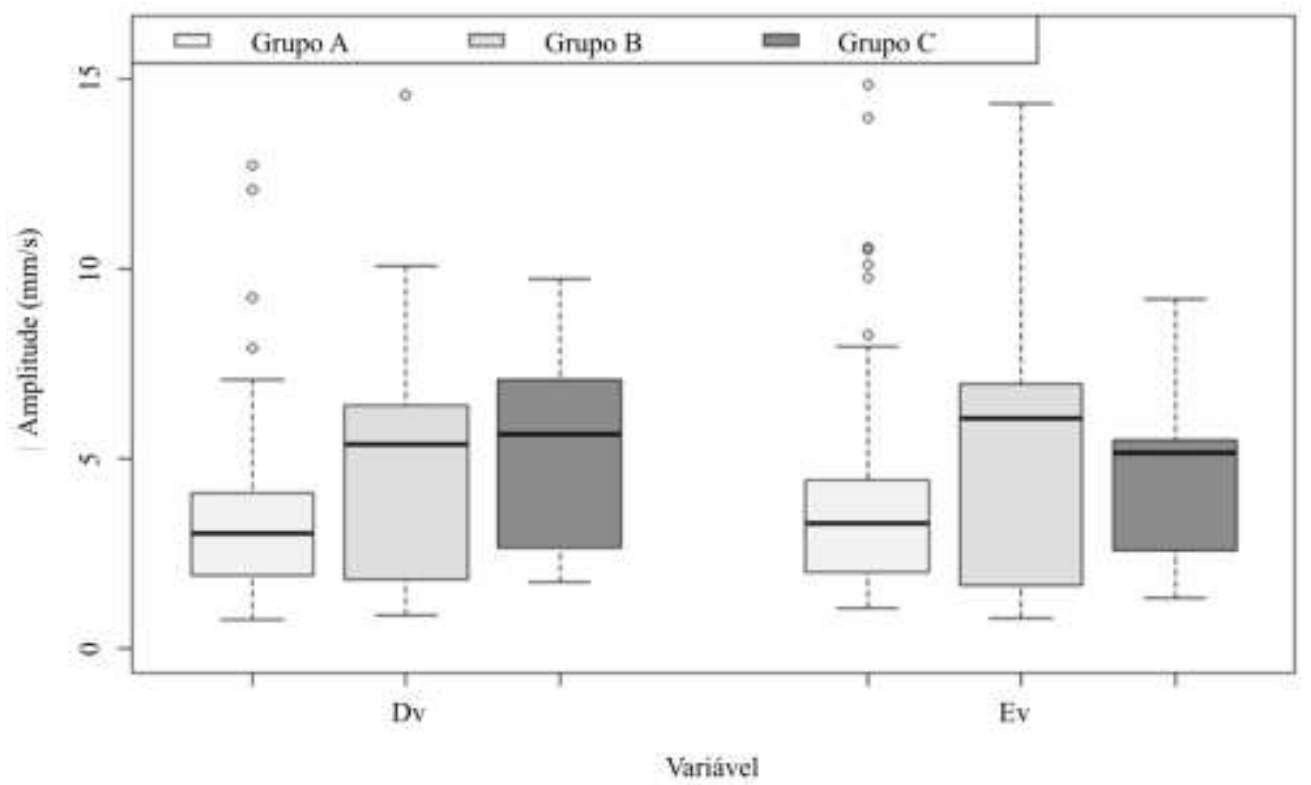

Fonte: Autores (2021).

As representações das Figuras 3-7 mostram que os dados obtidos trazem diferença na precisão motora entre os grupos em um índice de 100\% das variáveis, com variações com tendência de C > B > A em 87,5\% dos casos quando considerada a análise central dos dados. Os valores mais discriminantes foram observados nos dedos da mão direita, principalmente para o grupo C. Este grupo também representou o maior quantitativo de dados fora do padrão. O grupo B caracterizou-se como intermediário e o grupo A se manteve como o de menor desvio motor em 100\% dos membros.

Em termos gerais, estes dados mostram que as frequências de movimentação dos dedos (D1, D2, D3, D4, D5, E1, E2, E3, E4 e E5), de ambas as mãos, tiveram potencial de diferenciação entre os grupos. Entre as demais variáveis, a direção (DD e ED) apresenta-se como mais discriminante que a posição e a velocidade (DV e EV). Apesar da discreta diferença na maior parte das variáveis expostas nos gráficos em caixa, existem intersecções entre o terceiro quartil e o primeiro quartil subjacente, o que delimita uma percepção da distribuição das frequências de movimento entre os grupos.

\section{Discussão}

O LMC é um sensor de baixo custo, não invasivo e portátil, validado por Weichert et al. (2013) e Guna et al. (2014) e que possui índices de precisão maiores que outros sensores disponíveis no mercado, como Microsoft Kinect (Weichert et al., 2013). Os estudos de validação sugerem a sua aplicação em softwares voltados a medicina e engenharias, considerando seu alto desempenho. Os autores discutem que o dispositivo é apresenta maior custo-benefício em diversas aplicações, possuindo quedas de precisão apenas em situações em que objeto se afasta do sensor (ex.: $+250 \mathrm{~mm}$ em y) e que a queda de frequência pode ocorrer em situações precárias de captura, com valor médio de $40 \mathrm{~Hz}$ (Guna et al., 2014).

Embora haja uma diferença de visão entre os autores quanto ao quesito de aplicabilidade, a depender das condições em que se insere, ambos os trabalhos chegaram a valores de precisão sempre inferiores a $1 \mathrm{~cm}$. As variações com tendência de C > B > A em 87,5\% dos casos apresentados nesse estudo corroboram outros estudos que empregaram o sensor e obtiveram resultados promissores (Guzsvinecz, Szucs \& Sik-Lanyi, 2019; Kim, Park \& Lee, 2020; Colombini et al., 2021).

Outro estudo, desta vez de Vysocký et al. (2020), também realizou uma investigação dos atributos do sensor, destacando suas especificidades técnicas, benefícios e dando indicações de aplicação para a obtenção de dados confiáveis na 
caixa de interação do dispositivo. Nota-se a considerável parcela de trabalhos que utilizaram o LMC em pacientes com patologias do SNC, principalmente os acometidos por AVC, a exemplo de trabalhos recentes como de Wang et al. (2017), Cortés-Perez et al. (2021), Kim, Park \& Lee (2020).

Neste presente estudo, o LMC foi aplicado na obtenção de 16 pontos das mãos de cirróticos de forma minimamente invasiva e com finalidade de diferenciação motora entre diferentes níveis de complicação da doença por EH. Outros estudos também já aplicaram o dispositivo na quantificação da motricidade, em especial para a análise do tremor na doença de Parkinson (Butt et al., 2018; Vivar et al., 2019). Estes estudos utilizaram os dados com inteligência artificial e chegaram a resultados e conclusões diferentes. Butt et al. (2018) obteve resultados variando entre 70-80\% de acurácia, enquanto Vivar et al. (2019) chegou a resultados com $100 \%$ de acertos na diferenciação, considerando, inclusive, que suas técnicas de processamento de dados não foram iguais.

Nesta pesquisa, a inteligência artificial foi utilizada apenas para o balanceamento entre os grupos, causado pelo baixo quantitativo de pacientes com sinais aparentes de EH grau I e II durante o período de coleta, realizado em período de pandemia causada pelo SARS-Cov-2, o que dificulta a obtenção de uma análise real significativa com um tamanho de amostra de pacientes pequeno. Lizardo (2020) traz presente em um artigo de opinião algumas reflexões sobre esta problemática, as quais o presente trabalho acrescenta a ausência de pacientes nos ambulatórios para aqueles que realizam pesquisas clínicas.

Buscando superar esta problemática, foi realizado o balanceamento dos grupos e a consequente majoração da baixa amostragem foi realizada com a geração dos dados sintéticos, obtidos com SMOTE, que também foi aplicada em trabalhos relacionados à saúde pública nos mais diversos temas (Sui, Wei \& Zhao, 2015; Alghamdi et al., 2017; Turlapati \& Prusty, 2020; Teh et al., 2020). Não foram utilizadas técnicas para diferenciar ou prever a EH até este ponto de estudo.

Todavia, a aplicação dos testes estatísticos por si só e as visualizações de distribuição em gráficos de caixa pressupõem que existem diferenças motoras que tendem à elevação do desvio conforme a doença e o comprometimento hepático progride para maioria das variáveis observadas, o que corrobora com os estudos de Vilstrup et al. (2014). Os pontos de captura foram apresentados de forma isolada, não permitindo diferenciação entre os grupos por combinação.

Este trabalho foi desenvolvido com o cálculo tridimensional da distância entre dois pontos e os valores analisados foram as medianas, considerando a ausência de distribuição normal e homogeneidade. No entanto, outras técnicas de arranjos e análise dos dados serão ser aplicados aos dados e serão abordados em trabalhos futuros.

Este é o primeiro estudo que busca a classificação dos pacientes com EH pela motricidade, além de ter o benefício de ser realizado com a utilização de equipamentos de baixo custo e de forma minimamente invasiva. Trata-se, portanto, de um estudo piloto que, devido ao montante de dados gerados pela captura com o LMC, uma análise mais profunda pode e deve ser realizada por meio de algoritmos de inteligência artificial. O trabalho de investigação e obtenção de dados também deve seguir para o balanceamento e a majoração das classes, eliminando as necessidades de dados sintéticos, além da adição de um grupo composto por não cirróticos para fornecer um grupo controle apropriado para novas análises.

Outro ponto a ser considerado para as investigações que virão é a identificação de indivíduos com EH mínima e de pacientes que, de fato, não possuem EH. Isso será feito a partir da clusterização do grupo A. Estudos já abordaram a fase mínima investigando isoladamente com a aplicação de inteligência artificial. Esses trabalhos analisaram as redes de conectividade da atividade cerebral intrínseca e se apresentaram como possíveis sensores aliados à outras técnicas de identificação da patologia (Jiao, Teng \& Wang, 2013; Chen et al., 2016; Jiao et al. 2017).

\section{Conclusão}

Os resultados sugerem que é possível diferenciar as classes de pacientes e que a progressão do desvio motor segue como uma das complicações do agravamento da doença, um resultado já esperado e observado na prática médica cotidiana em 
conjunto com outros sinais evidentes da classificação de West-Haven.

\section{Agradecimentos}

Agradecimento aos profissionais do Serviço de Gastroenterologia e à Seção de Ensino, Aperfeiçoamento e Pesquisa do Hospital Geral de Fortaleza pelo apoio técnico e logístico na realização desta pesquisa.

\section{Referências}

Agarwal, R., \& Baid, R. A. (2016). Journal of Postgraduate Medicine. 62 (2). https://www.ncbi.nlm.nih.gov/pmc/articles/PMC4944342.

Alghamdi, M., Al-Mallah, M., Keteyian, S., Brawner, C., Ehrman, J., \& Sakr, S. (2017). Predicting diabetes mellitus using SMOTE and ensemble machine learning approach: the henry ford exercise testing (fit) project. Plos One. 12 (7); 179805. https://journals.plos.org/plosone/article?id=10.1371/journal.pone.0179805.

Amodio, P. (2018). Hepatic encephalopathy: Diagnosis and management. Liver Int. 38 (6); 966-75. https://onlinelibrary.wiley.com/doi/full/10.1111/liv.13752

Bajaj, J. S., et al (2008). Navigation skill impairment: another dimension of the driving difficulties in minimal hepatic encephalopathy. Hepatology. 47 (2); 596-604. https://aasldpubs.onlinelibrary.wiley.com/doi/full/10.1002/hep.22032.

Bittencout, P. L., et al (2011) Encefalopatia Hepática: Relatório da $1^{\text {a }}$ Reunião Monotemática da Sociedade Brasileira de Hepatologia. Gastroenterologia e Endoscopia Digestiva. 2011; 30 (nd); 10-34. http://sbhepatologia.org.br/pdf/encefalopatia/ged.pdf.

Butt, A. H., et al (2018). Objective and automatic classification of Parkinson disease with Leap Motion controller. Biomedical Engineering Online. 17 (1). https://biomedical-engineering-online.biomedcentral.com/articles/10.1186/s12938-018-0600-7.

Campagna, F., et al (2017). The animal naming test: an easy tool for the assessment of hepatic encephalopathy. Hepatology. 66 (1); 198218https://aasldpubs.onlinelibrary.wiley.com/doi/abs/10.1002/hep.29146.

Casula, M., Rangarajan N, \& Shields P. (2020). The potential of working hypotheses for deductive exploratory research. Qual Quant. 54 (5-6). https://link.springer.com/article/10.1007\%2Fs11135-020-01072-9

Chen, Q. F., et al (2016). Machine learning classification of cirrhotic patients with and without minimal hepatic encephalopathy based on regional homogeneity of intrinsic brain activity. Plos One. 11 (3); 1-15. https://journals.plos.org/plosone/article?id=10.1371/journal.pone.0151263.

Colombini, G., et al (2021). LEAP Motion Technology and Psychology: A Mini-Review on Hand Movements Sensing for Neurodevelopmental and Neurocognitive Disorders. Enviromental Research and Public Health. 18(8); 4006-4014; https://www-ncbi-nlmnih.ez68.periodicos.capes.gov.br/pmc/articles/PMC8069152/

Cortés-Pérez, I., et al (2021). Leap Motion Controller Video Game-Based Therapy for Upper Extremity Motor Recovery in Patients with Central Nervous System Diseases: A Systematic Review with Meta-Analysis. Sensors.21(6); 2065-70. https://www.mdpi.com/1424-8220/21/6/2065

Dzikri, A. I., \& Kurniawan, D. E. (2018). Hand Gesture Recognition for Game 3D Object Using The Leap Motion Controller with Backpropagation Method. 2018 International Conference On Applied Engineering (Icae). 1-5. https://ieexplore.ieee.org/document/8579400.

Guna, J., Jakus, G., Pogačnik, M., Tomažič, S., \& Sodnik, J. (2014). An analysis of the precision and reliability of the leap motion sensor and its suitability for static and dynamic tracking. Sensors. 14(2); 3702-20. https://www.mdpi.com/1424-8220/14/2/3702/htm

Guzsvinecz, T., Szucs, V., \& Sik-Lanyl, C. (2019). Suitability of the Kinect Sensor and Leap Motion Controller-A Literature Review. Sensors. 19 (5); 10721086.: https://www-ncbi-nlm-nih.ez68.periodicos.capes.gov.br/pmc/articles/PMC6427122/.

Jiao, Y., Teng, G., \& Wang, X. (2013). Predictive model for minimal hepatic encephalopathy based on cerebral functional connectivity. International Conference on Biomedical Engineering and Informatics. 6 (1); 541-45. https://www.ncbi.nlm.nih.gov/pmc/articles/PMC5599725.

Jiao, Y., et al (2017) Predictive models of minimal hepatic encephalopathy for cirrhotic patients based on large-scale brain intrinsic connectivity networks. Scientific Reports. 7(1); 1-12. https://www.nature.com/articles/s41598-017-11196-y

Kim, S., Park, S., \& Lee, O. (2020). Development of a Diagnosis and Evaluation System for Hemiplegic Patients Post-Stroke Based on Motion Recognition Tracking and Analysis of Wrist Joint Kinematics. Sensors. 20 (16); 4548. https://www.mdpi.com/1424-8220/20/16/4548.

Lizardo, J. L. (2020). O desafio de produzir uma pesquisa no meio de uma pandemia: o olhar de uma pesquisadora estrangeira no Brasil. Educação, Comunicação e Tecnologia. 2 (2), 25-33. https://revista.uemg.br/index.php/sciasedcomtec/article/view/5020.

Nardone, R., et al (2016). Minimal hepatic encephalopathy: A review. Neuroscience Research. 111(ni); 1-12. https://www.sciencedirect.com/science/article/abs/pii/S0168010216300499?via\%3Dihub.

Pinh,o M., Cerqueira R, \& Peixoto B. (2011). Pontuação psicométrica da encefalopatia hepática: Dados da normalização para a população portuguesa. Acta Médica Portuguesa. 24 (2); 319-26. https://www.actamedicaportuguesa.com/revista/index.php/amp/article/viewFile/1485/1071.

Piovesan, A., \& Temporini, E. R. (1995) Pesquisa exploratória: procedimento metodológico para o estudo de fatores humanos no campo da saúde pública. Rev. Saúde Pública. 29 (4); 318-325. Recuperado de: https://www.scielo.br/j/rsp/a/fF44L9rmXt8PVYLNvphJgTd/?lang=pt\&format=pdf. 
Research, Society and Development, v. 10, n. 7, e48310716749, 2021

(CC BY 4.0) | ISSN 2525-3409 | DOI: http://dx.doi.org/10.33448/rsd-v10i7.16749

Pires, M. R., \& Marinho, R. T. (2016). Impacto biopsicossocial da encefalopatia hepática. Dissertação de mestrado, Faculdade de Medicina, Universidade de Lisboa. https://repositorio.ul.pt/bitstream/10451/29101/1/MarianaRPires.pdf.

Sotil, E. U., et al (2009). The impact o preoperative overt encephalopathy on neurocognitive function after liver transplantation. Liver Transplant. 15 (2); $184-$ 192. https://aasldpubs.onlinelibrary.wiley.com/doi/full/10.1002/lt.21593.

Sui, Y., Wei, Y., \& Zhao, D. (2015). Computer-Aided Lung Nodule Recognition by SVM Classifier Based on Combination of Random Undersampling and SMOTE. Computational and Mathematical Methods In Medicine. 1-13. https://www.hindawi.com/journals/cmmm/2015/368674.

The, K., et al (2020). Imbalanced learning: improving classification of diabetic neuropathy from magnetic resonance imaging. Plos One. 15 (12); 243907. https://journals.plos.org/plosone/article?id=10.1371/journal.pone.0243907.

Turlapati, V. P. K., \& Prusty, M. R. (2020). Outlier-SMOTE: a refined oversampling technique for improved detection of covid-19. Intelligence-Based Medicine. 3(4); 100023. https://www.sciencedirect.com/science/article/pii/S2666521220300235?via\%3Dihub.

Vilstrup, H., et al (2014). Hepatic encephalopathy in chronic liver disease: 2014 Practice Guideline by the American Association for the Study of Liver Diseases and the European Association for the Study of the Liver. Hepatology. 60 (2); 715-35. http://www.clubepatologiospedalieri.it/wpcontent/uploads/2017/01/Linee-guida-AASLD-Encefalopatia-Epatica-2014.pdf.

Vivar, G., et al (2019). Contrast and Homogeneity Feature Analysis for Classifying Tremor Levels in Parkinson's Disease Patients. Sensors. 19 (9); 2072. https://www.mdpi.com/1424-8220/19/9/2072.

Vysocký, A., et al (2020). Analysis of Precision and Stability of Hand Tracking with Leap Motion Sensor. Sensors. 20 (15); 4088-93. https://www.mdpi.com/1424-8220/20/15/4088

Wang, Z., et al (2017). Leap Motion-based virtual reality training for improving motor functional recovery of upper limbs and neural reorganization in subacute stroke patients. Neural Regeneration Research. 12 (11); 1823-28. https://www.nrronline.org/article.asp?issn=16735374 ;year $=2017$; volume $=12 ;$ issue $=11$; spage $=1823$; epage $=1831$; aulast $=$ Wang .

Weichert, F., et al (2013). Analysis of the accuracy and robustness of the Leap Motion Controller. Sensors. 13 (5); 6380-93. https://www.mdpi.com/1424$8220 / 13 / 5 / 6380 / \mathrm{htm}$.

Wijdicks, E. F. M. Hepatic encephalopathy. New England Journal of Medicine. 2016; 375(17); 1660-70. https://www.nejm.org/doi/10.1056/NEJMra1600561. 